\title{
Testing the heteroscedastic error structure in quantile varying coefficient models
}

\author{
Irène Gijbels ${ }^{1}$, Mohammed A. Ibrahim ${ }^{2}$ and Anneleen Verhasselt ${ }^{2 *}$ \\ ${ }^{1}$ Department of Mathematics and Leuven Statistics Research Center (LStat), KU Leuven, Belgium. \\ ${ }^{2}$ Censtat, I-BioStat, Universiteit Hasselt, Belgium
}

Key words and phrases: Heteroscedasticity ; likelihood-ratio test ; penalized splines ; quantile regression ; varying coefficient models.

MSC 2010: Primary 62GXX; secondary 62G10; 62HXX

Abstract: In mean regression the characteristic of interest is the conditional mean of the response given the covariates. In quantile regression the aim is to estimate any quantile of the conditional distribution function. For given covariates the conditional quantile function fully characterizes the entire conditional distribution function, in contrast to the mean which is just one of its characteristic quantities. Regression quantiles substantially out-perform the least-squares estimator for a wide class of non-Gaussian error distributions. In this paper we consider quantile varying coefficient models (VCMs) that are an extension of classical quantile linear regression models, in which one allows the coefficients to depend on other variables.

We consider VCMs with various structures for the variance of the errors (the variability function) in order to allow for heteroscedasticity. For longitudinal data, the time $(T)$ dependent coefficient functions in the signal and the variability functions are estimated with P-splines (Penalized B-splines). Consistency of the proposed estimators is proved. Further, likelihood-ratio-type tests are considered for comparing the variability functions. The performance of the testing procedure is illustrated on simulated and real data. The Canadian Journal of Statistics xx: 1-32; 2017

(c) 2017 Statistical Society of Canada

(C) 2017 Statistical Society of Canada/Société statistique du Canada 
Résumé: Insérer votre résumé ici. We will supply a French abstract for those authors who can't prepare it themselves. La revue canadienne de statistique xx: 1-32; 2017 (c) 2017 Société statistique du Canada

\section{INTRODUCTION}

As an alternative to least squares regression, quantile regression allows a wide range of applications, where several conditional quantiles are of interest instead of just the conditional mean. Quantile regression does not assume a specific form for the (conditional) distribution. When the response, given the covariates, has a heavy-tailed distribution, quantile regression puts a reduced weight on the extreme observations.

Quantile regression as a generalization of median regression was first introduced by Koenker \& Bassett (1978). They considered a linear regression setting and introduced an estimation technique for the parameters by writing the optimization problem as a linear programming algorithm. The authors also derived asymptotic distributional results for the parameter estimates.

As a completely nonparametric relationship between a response variable $Y \in \mathbb{R}$ and several covariates $\left(T, X^{(1)}, \ldots, X^{(p)}\right)^{\prime} \in \mathbb{R}^{p+1}\left(\mathbf{A}^{\prime}\right.$ denotes the transpose of a vector or matrix A) is difficult to estimate and to alleviate the curse of dimensionality, varying coefficient models (VCMs) were introduced by Hastie \& Tibshirani (1993). VCMs are a useful generalization of linear regression models for depicting dynamic behaviors. Due to the flexibility and interpretability of VCMs, a lot of research has been done on parameter estimation and hypothesis testing, but mostly in a mean regression setting. VCMs are used to model longitudinal data (see e.g., Antoniadis, Gijbels, \& Verhasselt, 2012; Tang, Wang, \& Zhu, 2013; Andriyana, Gijbels, \& Verhasselt, 2014).

\footnotetext{
* Author to whom correspondence may be addressed.

E-mail: anneleen.verhasselt@uhasselt.be
} 
Several smoothing methods are considered in the literature for quantile regression: local polynomial in Chaudhuri (1991), Honda (2004) and Cai \& Xu (2008), smoothing splines in He, Ng, \& Portnoy (1998), B-splines in He \& Shi (1994) and Kim (2007) and penalized B-splines (Psplines) in Bollaerts, Eilers, \& Aerts (2006) and Andriyana, Gijbels, \& Verhasselt (2014, 2017). There is also a vast literature on variable selection in VCMs, when the focus is on quantile estimation (e.g., Noh, Chung, \& Van Keilegom, 2012; Tang, Wang, \& Zhu, 2013).

Quantile regression in VCMs using P-splines estimation is studied in Andriyana (2015). In that paper simple and general heteroscedastic error structures for the variance of the errors (the variability function) that are linear (in the parameters/covariates) are investigated. Here we consider more elaborate structures for the variability function, like power and exponential functions and prove that our estimator is consistent when the variability function is of an exponential type. Furthermore, we provide a test for choosing between two variability functions based on a likelihood-ratio-type test procedure.

The estimation of the conditional quantiles relies on a non-differentiable and asymmetric check loss function of Koenker \& Bassett (1978), $\rho_{\tau}(z)=z[\tau-I(z<0)]$ with $I(A)=1$ if $A$ holds, and 0 if not, rather than the square loss function (in mean regression). Therefore, the computational and theoretical aspects of conditional quantile regression are different to that of mean regression. For the estimation, linear programming is considered.

The rest of this paper is organized as follows. In Section 2, four different structures for the variability function are proposed. Sections 3 and 4 deal with estimation and inference methods of the variability functions, respectively. Simulation studies are carried out in Section 5. The estimation method and the testing procedure are applied to real data in Section 6. Finally, Section 7 presents a discussion and conclusions. Details of the proofs are deferred to the Appendix. This paper is subjoined with online Supplementary Material, which contains some information on practical implementation issues pertaining to the proposed method, further simulation results, an 
additional real data analysis as well as the $\mathrm{R}$ code of the procedure.

\section{HETEROSCEDASTIC MODEL}

We investigate quantile regression in VCMs with a heteroscedastic error structure. A longitudinal observational data scheme is considered, where each subject has measurements at different time points. The measurements from different subjects are assumed to be independent, but measurements at different time points within each subject can be correlated.

We have longitudinal observations $\left(Y\left(t_{i j}\right), \mathbf{X}^{*}\left(t_{i j}\right)^{\prime}, t_{i j}\right)^{\prime}$ of $\left(Y(T), \mathbf{X}^{*}(T)^{\prime}, T\right)^{\prime} \in \mathbb{R}^{p+2}$ with $\mathbf{X}^{*}(T)=\left(X^{(1)}(T), \ldots, X^{(p)}(T)\right)^{\prime}$ for $i=1, \ldots, n, j=1, \ldots, N_{i}$, and $p \geq 1$, where $t_{i j}$ is the $j$ th measurement time for the $i$ th subject, $N_{i}$ is the number of repeated measurements for the $i$ th subject, and $Y\left(t_{i j}\right)$ and $\mathbf{X}^{*}\left(t_{i j}\right)=\left(X^{(1)}\left(t_{i j}\right), \ldots, X^{(p)}\left(t_{i j}\right)\right)^{\prime}$ are the observed outcome and covariates of the $i$ th subject at time point $t_{i j}$. The time points $t_{i j}$ take values in the space $\mathcal{T} \subset \mathbb{R}$, $\mathbf{X}(T)=\left(X^{(0)}(T), X^{(1)}(T), \ldots, X^{(p)}(T)\right)^{\prime}$ with $X^{(0)}(T) \equiv 1$. We consider the VCM:

$$
\begin{aligned}
Y(T) & =\mathbf{X}^{\prime}(T) \boldsymbol{\beta}(T)+V(\mathbf{X}(T), T) \epsilon(T) \\
& =\beta_{0}(T)+\beta_{1}(T) X^{(1)}(T)+\ldots+\beta_{p}(T) X^{(p)}(T)+V(\mathbf{X}(T), T) \epsilon(T),
\end{aligned}
$$

where we have errors $\epsilon\left(t_{i j}\right)$ of $\epsilon(T) \in \mathbb{R}$ for $i=1, \ldots, n, j=1, \ldots, N_{i}$ and $\boldsymbol{\beta}(T)=$ $\left(\beta_{0}(T), \beta_{1}(T), \ldots, \beta_{p}(T)\right)^{\prime}$ is the vector of unknown regression coefficient functions at time $T$, with $\beta_{0}(T)$ the baseline effect. We call $V(\mathbf{X}(T), T)$ the variability function.

We estimate the $\tau$ th quantile of $Y(T), \beta_{k}(T)$ (with $k=0, \ldots, p$ ) and $V(\mathbf{X}(T), T)$ with the P-splines smoothing technique (here P-splines refers to penalized B-splines, that were introduced by Eilers \& Marx (1996)). The use of this smoothing technique is appealing here since it allows for flexible modeling and has computational advantages in VCMs.

In the mean regression case, Davidian \& Giltinan (1995) consider the square of the mean function for the error variance; Fortin et al. (2007) propose an unknown power of the mean 
function. Inspired by these proposals, we consider variability functions of the following form:

$$
V(\mathbf{X}(T), T)=\gamma(T) f_{\theta(T)}(\|\mathbf{X}(T)\|)
$$

where $\gamma(T) \geqslant 0, f_{\theta(T)}(\|\mathbf{X}(T)\|) \geqslant 0$, for a well-defined vector norm, and $\theta(T) \in \mathbb{R}$. More specifically, the following four special cases of the variability function $V(\mathbf{X}(T), T)$ in Equation (2) will be investigated in this paper (with $\|\mathbf{X}(T)\|_{1}=\left|X^{(0)}(T)\right|+\left|X^{(1)}(T)\right|+\ldots+$ $\left.\left|X^{(p)}(T)\right|\right)$ :

$$
\begin{aligned}
& V_{1}: V(\mathbf{X}(T), T)=\gamma\left(\|\mathbf{X}(T)\|_{1}\right)^{\theta}(\text { with } \gamma \geq 0 \text { and } \theta \in \mathbb{R}) ; \\
& V_{2}: V(\mathbf{X}(T), T)=\gamma(T)\left(\|\mathbf{X}(T)\|_{1}\right)^{\theta} ; \\
& V_{3}: V(\mathbf{X}(T), T)=\gamma(T) \exp \left(\theta\left\|\mathbf{X}^{*}(T)\right\|_{1}\right)=\gamma(T)\left[\exp \left(\left\|\mathbf{X}^{*}(T)\right\|_{1}\right)\right]^{\theta} ; \\
& V_{4}: V(\mathbf{X}(T), T)=\gamma(T) \exp \left(\theta_{1}(T)\left|X^{(1)}(T)\right|+\ldots+\theta_{p}(T)\left|X^{(p)}(T)\right|\right) .
\end{aligned}
$$

The first variability function is a special case of the second one for $\gamma(T)=\gamma$. Modeling structure $V_{3}$ is a generalization of $V_{1}$ and $V_{2}$, in the sense that a first order approximation (for $\left\|\mathbf{X}^{*}(T)\right\|_{1}$ close to zero) of the exponential function $\exp \left(\left\|\mathbf{X}^{*}(T)\right\|_{1}\right)$ leads to $\|\mathbf{X}(T)\|_{1}$. Modeling structure $V_{4}$ is a further generalization of $V_{3}$. We say that structure $V_{1}$ is nested in $V_{2}$ (denoted hereafter as $V_{1} \subseteq V_{2}$ ), $V_{2}$ is "nested" (in an approximative sense) in $V_{3}$, and $V_{3}$ is nested in $V_{4}$. Model $V_{2}$, with $\theta=0$, is considered by Andriyana, Gijbels, \& Verhasselt (2017). A variability function similar to $V_{4}$ is considered by Van Keilegom \& Wang (2010), with a partially linear structure. In $V_{4}$, we allow the variability function to depend on each covariate and each coefficient is allowed to vary with time $T$. In such a framework one can check the constancy of the varying coefficients or do variable selection. By Assumption H1 stated in the next section, the above variability functions have an influence on all quantiles other than the median. 


\section{ESTIMATION PROCEDURE}

The aforementioned variability functions are investigated using an approach similar to that of Andriyana, Gijbels, \& Verhasselt (2017). Such an approach consists of adapting the approach of He (1997) to the context of VCMs, and is therefore termed the 'Adaptive He (AHe) approach' in the literature. The advantage of this approach is two-fold: it avoids crossing of the quantile regression curves (when several quantiles are estimated) and allows estimation (and identification) of the variability function. Note that the variability function is only identifiable when extra assumptions are made on the error structure.

We impose the following assumptions on the error structure:

H1. The conditional median of the error term equals zero: $q_{0.5}(\epsilon(T) \mid \mathbf{X}(T), T)=0$, where $q_{0.5}(\cdot \mid \mathbf{X}(T), T)$ represents the conditional median or 0.5 -quantile.

H2. The conditional median of the natural logarithm of the absolute value of the error term equals zero: $q_{0.5}(\ln |\epsilon(T)| \mid \mathbf{X}(T), T)=0$.

Although the assumptions may appear strong, they are satisfied for a properly standardized error structure, as can be seen from Equation (5) in Section 5.

The estimation procedure then consists of the following three steps:

1. Assuming H1, the median function is estimated,

2. assuming $\mathbf{H 2}$, the variability function is estimated, and

3. the $\tau$ th quantile regression estimate is obtained, for various values of $\tau \in(0,1)$, using the estimates from the previous two steps. 


\subsection{Step 1: estimation of the median}

Under Assumption H1, the median function of the model in Equation (1) is given by:

$$
\begin{aligned}
q_{0.5}(Y(T) \mid \mathbf{X}(T), T) & =\mathbf{X}^{\prime}(T) \boldsymbol{\beta}(T)+V(\mathbf{X}(T), T) q_{0.5}(\epsilon(T) \mid \mathbf{X}(T), T) \\
& =\mathbf{X}^{\prime}(T) \boldsymbol{\beta}(T)
\end{aligned}
$$

The coefficient functions $\beta_{k}(T)$ can be approximated via normalized B-splines of degree $\nu_{k}$, for $k=0,1, \ldots, p$ :

$$
\begin{aligned}
\beta_{k}(T) & \approx \alpha_{k 1} B_{k 1}\left(T, \nu_{k}\right)+\alpha_{k 2} B_{k 2}\left(T, \nu_{k}\right)+\ldots+\alpha_{k m_{k}} B_{k m_{k}}\left(T, \nu_{k}\right) \\
& =\sum_{l=1}^{m_{k}} \alpha_{k l} B_{k l}\left(T, \nu_{k}\right)=\boldsymbol{\alpha}_{k}^{\prime} \mathbf{B}_{k}\left(T, \nu_{k}\right),
\end{aligned}
$$

where $\boldsymbol{\alpha}_{k}=\left(\alpha_{k 1}, \ldots, \alpha_{k m_{k}}\right)^{\prime}$ denotes the coefficient vector of the associated B-splines $\mathbf{B}_{k}\left(T, \nu_{k}\right)=\left(B_{k 1}\left(T, \nu_{k}\right), \ldots, B_{k m_{k}}\left(T, \nu_{k}\right)\right)^{\prime}, m_{k}=u_{k}+\nu_{k}$, and $u_{k}$ represents the number of equidistant knots in the B-splines approximation of the $k$ th component $\left(X^{(k)}(T)\right)$. A thorough discussion on the choice of the number of knots $u_{k}$ and the degree of the splines $\nu_{k}$ can be found in Gijbels \& Verhasselt (2010). The coefficient function $\beta_{k}(T)$ is estimated by,

$$
\hat{\beta}_{k}(T)=\hat{\boldsymbol{\alpha}}_{k}^{\prime} \mathbf{B}_{k}\left(T, \nu_{k}\right)
$$

where $\hat{\boldsymbol{\alpha}}_{k}=\left(\hat{\alpha}_{k 1}, \ldots, \hat{\alpha}_{k m_{k}}\right)^{\prime}(k=0, \ldots, p)$, and $\hat{\boldsymbol{\alpha}}=\left(\hat{\boldsymbol{\alpha}}_{0}^{\prime}, \ldots, \hat{\boldsymbol{\alpha}}_{p}^{\prime}\right)^{\prime}$ is obtained by minimizing the following P-splines objective function, using an $L_{1}$-type of penalty function (Andriyana, Gijbels, \& Verhasselt, 2014):

$$
\sum_{i=1}^{n} \frac{1}{N_{i}} \sum_{j=1}^{N_{i}} \rho_{0.5}\left(Y\left(t_{i j}\right)-\sum_{k=0}^{p} \sum_{l=1}^{m_{k}} \alpha_{k l} B_{k l}\left(t_{i j}, \nu_{k}\right) X^{(k)}\left(t_{i j}\right)\right)+\sum_{k=0}^{p} \sum_{l=d_{k}+1}^{m_{k}} \lambda_{k}\left|\Delta^{d_{k}} \alpha_{k l}\right|
$$

with respect to $\boldsymbol{\alpha}=\left(\boldsymbol{\alpha}_{0}^{\prime}, \ldots, \boldsymbol{\alpha}_{p}^{\prime}\right)^{\prime}$, where $\lambda_{k}>0$ is the smoothing parameter for the $k$ th component; $d_{k}$ is the differencing order in the penalty term; and $\Delta^{d_{k}}$ is the $d_{k}$ th order differencing operator of the $k$ th variable: $\Delta^{d_{k}} \alpha_{k l}=\sum_{t=0}^{d_{k}}(-1)^{t}\left(\begin{array}{c}d_{k} \\ t\end{array}\right) \alpha_{k(l-t)}$. 


\subsection{Step 2: estimation of the variability function}

From the model in Equation (1) and since $V(\mathbf{X}(T), T) \geq 0$, we have

$$
\ln \left|Y(T)-\mathbf{X}^{\prime}(T) \boldsymbol{\beta}(T)\right|=\ln V(\mathbf{X}(T), T)+\ln |\epsilon(T)|
$$

Using Assumption H2, since for a monotone function $g($.$) the quantiles of the transformed ran-$ dom variable $g(Y)$ are simply the transformed quantiles of the original variable $Y$ (Koenker, 2005), we estimate $V(.,$.$) based on the following equation:$

$$
q_{0.5}\left(\ln \left|Y(T)-\mathbf{X}^{\prime}(T) \boldsymbol{\beta}(T)\right| \mid \mathbf{X}(T), T\right)=\ln V(\mathbf{X}(T), T)
$$

We now illustrate the estimation of the variability function under model $V_{2}$. First, under $V_{2}$, the conditional median of $\ln \left|Y(T)-\mathbf{X}^{\prime}(T) \boldsymbol{\beta}(T)\right|$ is given by:

$$
\begin{aligned}
q_{0.5}\left(\ln \left|Y(T)-\mathbf{X}^{\prime}(T) \boldsymbol{\beta}(T)\right| \mid \mathbf{X}(T), T\right) & =\ln \{\gamma(T)\}+\theta \ln \left\{\|\mathbf{X}(T)\|_{1}\right\} \\
& \approx \sum_{l=1}^{m^{\mathrm{v}}} \alpha_{l}^{\mathrm{v}} B_{l}^{\mathrm{v}}\left(T, \nu^{\mathrm{v}}\right)+\theta \ln \left\{\|\mathbf{X}(T)\|_{1}\right\}
\end{aligned}
$$

where $\ln \{\gamma(T)\}$ is approximated on a basis (of size $m^{\mathrm{v}}=u^{\mathrm{v}}+\nu^{\mathrm{v}}$ ) of normalized B-splines of degree $\nu^{\mathrm{v}}$ with $u^{\mathrm{v}}+1$ equidistant knots.

Note that this approximation can be done in a similar fashion for all other modeling structures for the variability function, where smooth functions in $T$ are approximated with B-splines.

The parameter estimates for the variability structure under $V_{2}$ are obtained by solving:

$$
\begin{array}{r}
\min _{\alpha_{1}^{\mathrm{v}}, \alpha_{2}^{\mathrm{v}}, \ldots, \alpha_{m \mathrm{v}}^{\mathrm{v}}, \theta}\left\{\sum _ { i = 1 } ^ { n } \frac { 1 } { N _ { i } } \sum _ { j = 1 } ^ { N _ { i } } \rho _ { 0 . 5 } \left(\ln \left(\left|Y\left(t_{i j}\right)-\mathbf{X}^{\prime}\left(t_{i j}\right) \hat{\boldsymbol{\beta}}\left(t_{i j}\right)\right|\right)-\sum_{l=1}^{m^{\mathrm{v}}} \alpha_{l}^{\mathrm{v}} B_{l}^{\mathrm{v}}\left(t_{i j}, \nu^{\mathrm{v}}\right)\right.\right. \\
\left.\left.-\theta \ln \left\{\left\|\mathbf{X}\left(t_{i j}\right)\right\|_{1}\right\}\right)+\lambda^{\mathrm{v}} \sum_{l=d^{\mathrm{v}}+1}^{m^{\mathrm{v}}}\left|\Delta^{d^{\mathrm{v}}} \alpha_{l}^{\mathrm{v}}\right|\right\},
\end{array}
$$


where $\lambda^{v}>0$ is the smoothing parameter and $d^{v}$ is the differencing order in the penalty term. The optimization problems for the other modeling structures are obtained in a similar way and omitted here for brevity.

Finally, the variability function under modeling structure $V_{2}$ is estimated by:

$$
\hat{V}(\mathbf{X}(T), T)=\hat{\gamma}(T)\|\mathbf{X}(T)\|_{1}^{\hat{\theta}}=\exp \left\{\sum_{l=1}^{m^{\mathrm{v}}} \hat{\alpha}_{l}^{\mathrm{v}} B_{l}^{\mathrm{v}}\left(T, \nu^{\mathrm{v}}\right)\right\}\|\mathbf{X}(T)\|_{1}^{\hat{\theta}} .
$$

Note that if one is only interested in estimating the conditional median of $Y$, one can stop after Step 1. However, if one is interested in other quantiles, the variability function and the conditional quantile of $\epsilon$ also have to be estimated.

\subsection{Step 3: estimation of $\tau$ th quantile}

The model in Equation (1) yields

$$
q_{\tau}\left(Y(T)-\mathbf{X}^{\prime}(T) \boldsymbol{\beta}(T) \mid \mathbf{X}(T), T\right)=V(\mathbf{X}(T), T) q_{\tau}(\epsilon(T) \mid \mathbf{X}(T), T) .
$$

Using the estimators $\hat{\boldsymbol{\beta}}(T)$ and $\hat{V}(\mathbf{X}(T), T)$ from Steps 1 and 2, respectively, we create a pseudoresponse $Y(T)-\mathbf{X}^{\prime}(T) \hat{\boldsymbol{\beta}}(T)$ and a pseudo-covariate $\hat{V}(\mathbf{X}(T), T)$.

We approximate the unknown conditional $\tau$ th quantile of the error term $\epsilon(T)$, denoted as $a^{\tau}(T)=q_{\tau}(\epsilon(T) \mid \mathbf{X}(T), T)$, by B-spline basis functions of degree $\nu^{q}$ with $u^{q}+1$ equidistant knots, leading to $a^{\tau}(T) \approx \sum_{l=1}^{m^{q}} \alpha_{l}^{q} B_{l}^{q}\left(T, \nu^{q}\right)$. We hereby also assume that $a^{\tau}(T)$ satisfies the Hölder condition: $a^{\tau}(T) \in \mathcal{H}_{r^{q}}$ for some $r^{q}>1 / 2$ (see Definition 2 in Appendix A). The coefficients $\left(\alpha_{1}^{q}, \alpha_{2}^{q}, \ldots, \alpha_{m^{q}}^{q}\right)$ are estimated by minimizing the following P-splines objective function:

$$
\begin{aligned}
\sum_{i=1}^{n} \frac{1}{N_{i}} \sum_{j=1}^{N_{i}} \rho_{\tau}\left(Y\left(t_{i j}\right)-\mathbf{X}^{\prime}\left(t_{i j}\right) \hat{\boldsymbol{\beta}}\left(t_{i j}\right)-\hat{V}\left(\mathbf{X}\left(t_{i j}\right), t_{i j}\right)\right. & \left.\sum_{l=1}^{m^{q}} \alpha_{l}^{q} B_{l}^{q}\left(t_{i j}, \nu^{q}\right)\right) \\
& +\sum_{l=d^{q}+1}^{m^{q}} \lambda^{q}\left|\Delta^{d^{q}} \alpha_{l}^{q}\right|
\end{aligned}
$$


with respect to $\left(\alpha_{1}^{q}, \alpha_{2}^{q}, \ldots, \alpha_{m^{q}}^{q}\right)$, where $\lambda^{q}>0$ is the smoothing parameter, and $d^{q}$ is the differencing order.

The $\tau$ th quantile of the error $a^{\tau}(T)$ is then estimated by: $\hat{a}^{\tau}(T)=\sum_{l=1}^{m^{q}} \hat{\alpha}_{l}^{q} B_{l}^{q}\left(T, \nu^{q}\right)$. Finally, the estimated conditional quantile function of the response $Y(T)$ is given by:

$$
\hat{q}_{\tau}(Y(T) \mid \mathbf{X}(T), T)=\mathbf{X}^{\prime}(T) \hat{\boldsymbol{\beta}}(T)+\hat{V}(\mathbf{X}(T), T) \hat{a}^{\tau}(T)
$$

\subsection{Consistency of the estimated variability function for $V_{4}$}

In this section we investigate the consistency of the proposed estimation procedure for $V_{4}$. We show that the P-splines quantile estimator for $V_{4}$ converges in $L_{2}$-norm to the unknown quantile function $q_{0.5}\left(\ln \left|Y(T)-\mathbf{X}^{\prime}(T) \boldsymbol{\beta}(T)\right| \mid \mathbf{X}(T), T\right)=\sum_{k=0}^{p} \theta_{k}(T)\left|X^{(k)}(T)\right|$, by proving that each $\hat{\theta}_{k}(T)$ converges in $L_{2}$-norm to the function $\theta_{k}(T)$.

The following theorem, taken from Theorem 2.1 in Andriyana, Gijbels, \& Verhasselt (2014), states that each individual estimate $\hat{\beta}_{k}$ (in the signal part of the model), defined in Equation (3), converges in $L_{2}$-norm to the regression coefficient function $\beta_{k}$ for an increasing number of knots. The definitions and assumptions are stated in Appendix A.

Theorem 1 Assume B1 - B7 hold. Furthermore, assume that $u_{\max }^{3 / 4} \lambda_{\max } n^{-1 / 2} \rightarrow 0$ as $n$ tends to $\infty$, where $u_{\max }=\max \left(u_{0}, \ldots, u_{p}\right)$ and $\lambda_{\max }=\max \left(\lambda_{0}, \ldots, \lambda_{p}\right)$. Then, $\| \hat{\beta}_{k}-$ $\beta_{k} \|_{2}=O_{p}\left(n^{-r_{\min } /\left(2 r_{\min }+1\right)}\right)$, where for any function $\beta_{k}:\left\|\beta_{k}\right\|_{2}^{2}=\int_{\mathcal{T}} \beta_{k}(t)^{2} d t$ and $r_{\min }=$ $\min \left\{r_{0}, \ldots, r_{p}\right\}$, which is the minimum of the Hölder constants of the $\beta_{k}$, defined in Definition 2. 
In the second step of our procedure we assume that $\ln |\epsilon(T)|$ has median zero, given $(\mathbf{X}(T), T)$. Note that relying on Theorem 1, we have:

$$
\begin{aligned}
\ln \left|Y(T)-\mathbf{X}^{\prime}(T)\{\boldsymbol{\beta}(T)+[\hat{\boldsymbol{\beta}}(T)-\boldsymbol{\beta}(T)]\}\right| \\
=\ln \left|Y(T)-\mathbf{X}^{\prime}(T)\left\{\boldsymbol{\beta}(T)+O_{p}\left(n^{-r_{\min } /\left(2 r_{\min }+1\right)}\right) \mathbf{1}_{p+1}\right\}\right| \\
\quad \stackrel{P}{\longrightarrow} \ln \left|Y(T)-\mathbf{X}^{\prime}(T) \boldsymbol{\beta}(T)\right|, \quad \text { as } n \rightarrow \infty
\end{aligned}
$$

where $\mathbf{1}_{p+1}$ is the vector of ones of dimension $p+1$.

Our main result on the consistency of the $\theta_{k}$, in the variability part of the model, follows. The proof is provided in Appendix C.

Theorem 2 Assume B6, B7, C1 - C6 hold. Furthermore, assume that $\left(u_{\max }^{V}\right)^{3 / 4} \lambda_{\max }^{V} n^{-1 / 2} \rightarrow 0$ as $n$ tends to $\infty$, where $u_{\max }^{V}=\max \left(u_{0}^{V}, \ldots, u_{p}^{V}\right)$ and $\lambda_{\max }^{V}=$ $\max \left(\lambda_{0}^{V}, \ldots, \lambda_{p}^{V}\right)$. Then,

$$
\left\|\hat{\theta}_{k}^{(j)}-\theta_{k}^{(j)}\right\|_{2}=O_{p}\left(n^{-\left(r_{\min }^{V}-j\right) /\left(2 r_{\min }^{V}+1\right)}\right), \quad j=0,1, \ldots, \nu_{k}^{V}-1
$$

where $\theta_{k}^{(j)}$ is the jth order derivative of $\theta_{k}$ and $r_{\min }^{V}=\min \left\{r_{0}^{V}, \ldots, r_{p}^{v}\right\}$, where $r_{k}^{v}$ is the Hölder constant of the function $\theta_{k}$ as in Condition $\mathbf{C 1 .}$

The convergence rates in Theorem 2 attain the optimal global rates established by Stone (1982) for non-parametric regression with independent and identically distributed data. Indeed if $\theta_{k}$ has bounded second order derivative and splines of at most degree 3 are used, the convergence rate is $n^{-2 / 5}$.

The proof of Theorem 2 is based on the fact that the number of knots does not grow too fast with sample size (see for example Assumption C3). This is similar to the approach of Claeskens, Krivobokova, \& Opsomer (2009) which leads to a scenario close to regression splines. 
The implementation and the choice of the smoothing parameters are deferred to Section S1 of the Supplementary Material.

\section{MODEL ASSESSMENT}

We now come to testing for a specific shape of the variability function.

\subsection{Variability function}

The following hypothesis tests are considered:

$$
\begin{aligned}
& H_{0}: V(\mathbf{X}(T), T) \text { in } V_{j} \text { versus } \\
& H_{1}: V(\mathbf{X}(T), T) \text { in } V_{k}
\end{aligned}
$$

where $V_{j} \subset V_{k}$.

We use the likelihood-ratio-type test considered by Kim (2007). The test statistic is defined as:

$$
\begin{aligned}
G=2\left\{\sum_{i=1}^{n} \frac{1}{N_{i}} \sum_{j=1}^{N_{i}} \rho_{0.5}\left(\left|Y\left(t_{i j}\right)-\mathbf{X}^{\prime}\left(t_{i j}\right) \hat{\boldsymbol{\beta}}\left(t_{i j}\right)\right|-\hat{m}_{0}\right)-\right. \\
\left.\sum_{i=1}^{n} \frac{1}{N_{i}} \sum_{j=1}^{N_{i}} \rho_{0.5}\left(\left|Y\left(t_{i j}\right)-\mathbf{X}^{\prime}\left(t_{i j}\right) \hat{\boldsymbol{\beta}}\left(t_{i j}\right)\right|-\hat{m}_{1}\right)\right\},
\end{aligned}
$$

where $\hat{m}_{0}$ and $\hat{m}_{1}$ are the estimated variability functions under $H_{0}$ and under $H_{1}$, respectively. It is expected that $\rho_{0.5}\left(\left|Y\left(t_{i j}\right)-\mathbf{X}^{\prime}\left(t_{i j}\right) \hat{\boldsymbol{\beta}}\left(t_{i j}\right)\right|-\hat{m}_{0}\right)$ is larger than $\rho_{0.5}\left(\left|Y\left(t_{i j}\right)-\mathbf{X}^{\prime}\left(t_{i j}\right) \hat{\boldsymbol{\beta}}\left(t_{i j}\right)\right|-\hat{m}_{1}\right)$, when $H_{0}$ is false. Therefore, with test statistic $G$, we assess the generality of the variability function. We consider four structures of variability function ( $V_{1}$ to $V_{4}$ of Section 2 ), as well as:

$V_{0}: V(\mathbf{X}(T), T)=\gamma($ homoscedastic model);

$V_{5}: V(\mathbf{X}(T), T)=\gamma(T)$ (the simple heteroscedastic model). 
Note that these six variability structures are "nested" in the following sense:

$$
V_{0} \subseteq\left(V_{1}, V_{5}\right) \subseteq V_{2} \underset{\sim}{\sim} V_{3} \subseteq V_{4}
$$

where $\underset{\sim}{\subset}$ means that $V_{3}$ is a generalization of $V_{2}$ in the sense that a first order approximation of the exponential function $\exp \left(\left\|\mathbf{X}^{*}(T)\right\|_{1}\right)$ leads to $\|\mathbf{X}(T)\|_{1}$. So, $V_{4}$ is considered the "full model" here.

The null hypothesis is rejected when $G$ is too large. The p-value is obtained using a resampling subject bootstrap. Since the data are longitudinal in nature and it is important to retain the dependence structure within a subject, the observations in each subject are treated as blocks.

1. Resample $n$ subjects with replacement from $i=1, \ldots, n$, to obtain the bootstrap sample $\left\{\left(Y^{b}\left(t_{i j}^{b}\right), \mathbf{X}^{b}\left(t_{i j}^{b}\right), t_{i j}^{b}\right): i=1, \ldots, n, j=1, \ldots, N_{i}^{b}\right\}$ from $\left\{\left(Y^{p}\left(t_{i j}\right), \mathbf{X}\left(t_{i j}\right), t_{i j}\right): i=\right.$ $\left.1, \ldots, n, j=1, \ldots, N_{i}\right\}$, with

$$
\begin{aligned}
Y^{p}\left(t_{i j}\right) & =\sum_{k=0}^{p} \hat{\beta}_{k}\left(t_{i j}\right) X^{(k)}\left(t_{i j}\right)+\hat{m}_{0} \epsilon^{p}\left(t_{i j}\right), \\
\epsilon^{p}\left(t_{i j}\right) & =\frac{Y\left(t_{i j}\right)-\sum_{k=0}^{p} \hat{\beta}_{k}\left(t_{i j}\right) X^{(k)}\left(t_{i j}\right)}{\hat{m}_{4}},
\end{aligned}
$$

where $\hat{m}_{0}$ and $\hat{m}_{4}$ are the estimated variability functions under $H_{0}$ and under the full model $\left(V_{4}\right)$, respectively.

2. Repeat the above sampling procedure $B$ times, where $B$ is the number of bootstrap replications.

3. Obtain the test statistic $G^{b}$ for each bootstrap sample to obtain its empirical distribution.

4. Get the p-value using the empirical probability of $G^{b} \geq G$.

The above testing procedure is implemented in the $\mathrm{R}$ software. The $\mathrm{R}$ code is also available in the Supplementary Material.

DOI:

The Canadian Journal of Statistics / La revue canadienne de statistique 


\subsection{Extensions}

$V_{4}$ is the most general model for the variability function considered here. It has a varying coefficient for each covariate $X^{(1)}, \ldots, X^{(p)}$. Hence, it can be of interest to investigate whether the coefficients are constant (or even not significant). This again can be tested in a similar way to that presented in Section 4.1, using the likelihood-ratio-type test, by putting the reduced model (where the varying coefficients are assumed constant or not significant) in $H_{0}$ and the full model $\left(V_{4}\right)$ in $H_{1}$.

\section{SIMULATION STUDY}

In this section we conduct simulation studies to compare the various variability structures discussed in the previous section. Four simulation settings, defined in Table 1, are used assuming the variability function structures $V_{1}, V_{2}, V_{3}$ and $V_{4}$ described in Section 4.1. These are referred to as Settings 1, 2, 3 and 4 here, with $\theta=2$. The results for Settings 2 and 3 are deferred to the Supplementary Material. We also consider, in the Supplementary Material, a simulation setting, called Setting 5, which combines Settings 1 and 2 to check the robustness of our testing procedure.

In order to ensure that the estimation tasks are of comparable difficulty in the four simulation settings, the coefficient functions are such that the signal to noise ratio (SNR) is approximately seven (as in Andriyana \& Gijbels, 2017) in each setting:

$$
S N R=\frac{\text { Sample variance of } \sum_{k=0}^{3} \beta_{k}(T) X^{(k)}(T)}{\text { Sample variance of } V(\mathbf{X}(T), T) \epsilon(T)} \approx 7 \text {. }
$$

The error term is generated from a transformed multivariate normal distribution to ensure that $\mathbf{H 1}$ and $\mathbf{H 2}$ hold. First, for $i=1, \ldots, n$, we generate $\left(\zeta\left(t_{i 1}\right), \ldots, \zeta\left(t_{i N_{i}}\right)\right)$ from $N(\mathbf{0}, \mathbf{C})$, a multivariate normal with zero mean vector and covariance matrix $\mathbf{C}$, whose element on the $j$ th row and $z$ th column is given by $\left.\operatorname{cov}\left(\zeta\left(t_{i j}\right), \zeta\left(t_{i z}\right)\right)=30 \exp (-|j-z|)\right)$. Then, the error term $\epsilon(T)$ 
TABLE 1: The description of the coefficients.

\begin{tabular}{lllll} 
Coefficients & Setting 1 & Setting 2 & Setting 3 & Setting 4 \\
& & & & \\
& & & & \\
$\beta_{0}(T)$ & $2 \sqrt{T}$ & $2 \sqrt{T}$ & $2 \sqrt{T}$ & $2 \sqrt{T}$ \\
$\beta_{1}(T)$ & $\frac{2}{5}(\pi T+10)$ & $\frac{5}{4}(\pi T+10)$ & $\pi T+10$ & $\frac{5}{4}(\pi T+10)$ \\
$\beta_{2}(T)$ & $\sin (\pi T / 30)+3$ & $\sin (\pi T / 30)+3$ & $\sin (\pi T / 30)+3$ & $\sin (\pi T / 30)+3$ \\
$\beta_{3}(T)$ & $\frac{(20-T)^{2}}{1000}-4$ & $(20-T)^{2}-4$ & $(20-T)^{2}-4$ & $\frac{(20-T)^{2}}{1000}-4$ \\
$\gamma(T)$ & 1 & $T / 4$ & $T / 8$ & $\beta_{0}(T) / 8$ \\
$\theta_{1}(T)$ & & & & $\sqrt{\beta_{1}(T)} / 2$ \\
$\theta_{2}(T)$ & & & & $\beta_{2}(T) / 50$ \\
$\theta_{3}(T)$ & & & & $\beta_{3}(T) / 50$ \\
\hline
\end{tabular}

is obtained from the following transformation,

$$
\epsilon\left(t_{i j}\right)=\frac{\zeta\left(t_{i j}\right)-q_{0.5}\left(\zeta\left(t_{i j}\right)\right)}{q_{0.5}\left(\left|\zeta\left(t_{i j}\right)-q_{0.5}\left(\zeta\left(t_{i j}\right)\right)\right|\right)} .
$$

The covariate $X^{(1)}(T)$ is generated from a standard exponential distribution, $X^{(2)}(T)$ is generated from a standard normal distribution, and $X^{(3)}(T)$ is generated from a uniform distribution $U[-1,1]$. The covariates are standardized in the following way:

$$
X^{(k)}(T)=\frac{X^{(k)}(T)-\min \left(X^{(k)}(T)\right)}{3\left\{\max \left(X^{(k)}(T)\right)-\min \left(X^{(k)}(T)\right)\right\}} .
$$

This standardization is done to ensure that the exponential function $\exp \left(\left\|\mathbf{X}^{*}(T)\right\|_{1}\right)$ is well approximated by its first order approximation $\|\mathbf{X}(T)\|_{1}$. We simulate 100 data sets with $n=100$ from each of the above settings. The time variable ranges from 0 to 49 . For each case $i$, the DOI: 
probability of having a measurement in each time point is 0.6 , creating an unbalanced number of measurements. The actual time points are calculated by adding a generated value from a $U[0,0.5]$ to the non-skipped time points.

To analyze the data sets B-splines of degree three with 11 equidistant knots in the time interval and differencing order 1 are used for each simulation setting. To determine the individual smoothing parameters, $\lambda_{k}$ 's, a Schwarz information criterion is used; details are presented in Section S1.2 of the Supplementary Material.

As discussed in Section 4.1, the six variability models are compared using likelihood-ratiotype hypothesis testing based on bootstrap resampling with $B=200$ bootstrap samples for all 100 simulated data sets. Proportions of significant tests are presented using 5\% level of significance.

\subsection{Setting 1}

It is expected, under this setting, that the estimated variability structure assuming $V_{1}$ performs better than or equivalently to $V_{0}$ and $V_{5}$, and has an equivalent performance to $V_{2}, V_{3}$ and $V_{4}$. Figure 1 (b) shows the root approximate integrated square error (RAISE), for the six aforementioned models, defined by

$$
\operatorname{RAISE}\left(\hat{V}^{(s)}(., .)\right)=\left(\sum_{i=1}^{n} \frac{1}{N_{i}} \sum_{j=1}^{N_{i}}\left[\hat{V}^{(s)}\left(\mathbf{X}\left(t_{i j}\right), t_{i j}\right)-V^{(s)}\left(\mathbf{X}\left(t_{i j}\right), t_{i j}\right)\right]^{2}\right)^{\frac{1}{2}}
$$

for simulation $s$.

In all figures we refer to a model with structure $V_{j}$ as "Model $\mathrm{j}$ ".

It can be seen from Figure 1 (a) that $V_{0}$ and $V_{5}$ have poorer performance than the other models. This is also observed from Figure 1 (b), the root approximate integrated square error of 


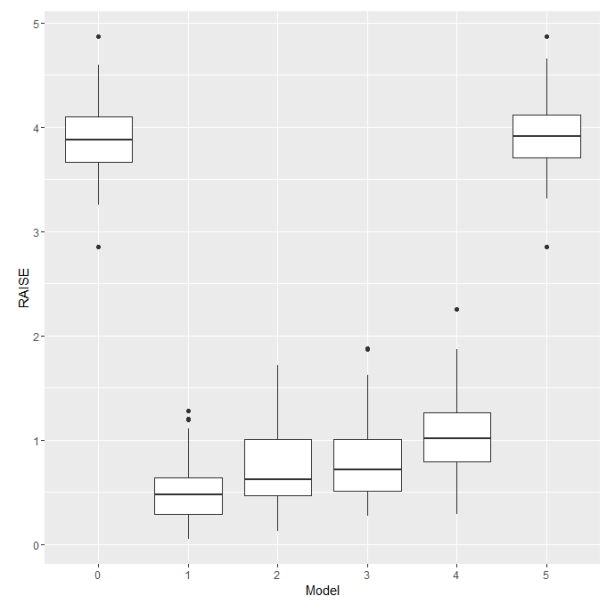

(a)

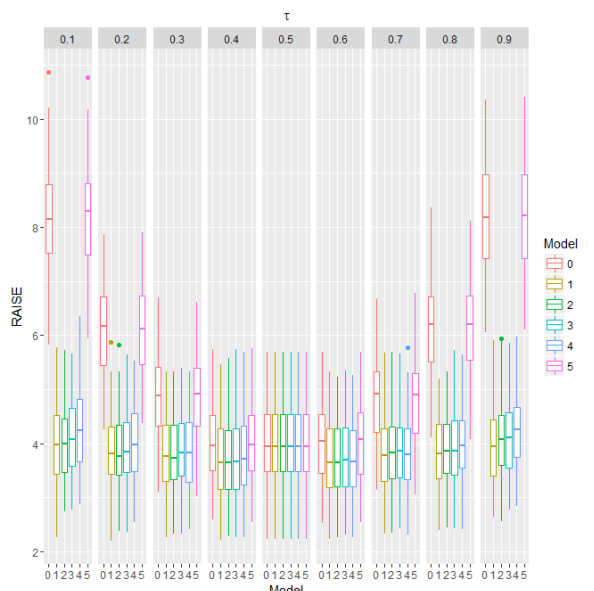

(b)

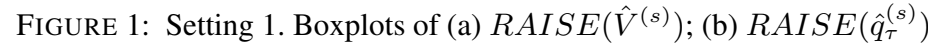

the quantiles from all simulated data sets, defined by

$$
\operatorname{RAISE}\left(\hat{q}_{\tau}^{(s)}(.)\right)=\left(\sum_{i=1}^{n} \frac{1}{N_{i}} \sum_{j=1}^{N_{i}}\left[\hat{q}_{\tau}^{(s)}\left(Y\left(t_{i j}\right) \mid \mathbf{X}\left(t_{i j}\right), t_{i j}\right)-q_{\tau}^{(s)}\left(Y\left(t_{i j}\right) \mid \mathbf{X}\left(t_{i j}\right), t_{i j}\right)\right]^{2}\right)^{\frac{1}{2}}
$$

for simulation $s$.

The true $V_{1}$ (Figure 2 (a)) as well as the estimated variability function (Figure 2 (b)) are presented for the sample associated with a 50th percentile performance in terms of $\operatorname{RAISE}\left(\hat{V}^{(s)}(.,).\right)$. It is observed that the estimated variability function of $V_{1}$ mimics the true variability function. The true and estimated quantiles evaluated at the maximum values of the covariates, using $V_{1}$, are presented in Figures 2 (c) and (d), respectively. As can be observed from these figures, $V_{1}$ mimics the true quantiles quite well.

Several comparisons of the models using the likelihood-ratio-type test are presented in Table 2. The hypothesis for the first testing procedure is:

$$
\begin{aligned}
& H_{0}: V(\mathbf{X}(T), T) \text { in } V_{0} \text { versus } \\
& H_{1}: V(\mathbf{X}(T), T) \text { in } V_{1} .
\end{aligned}
$$




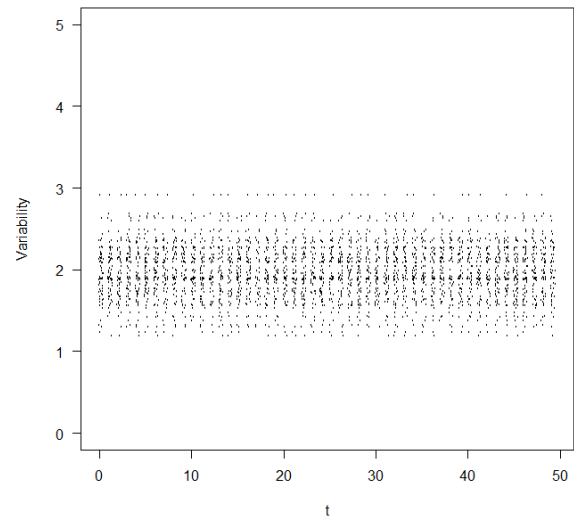

(a)

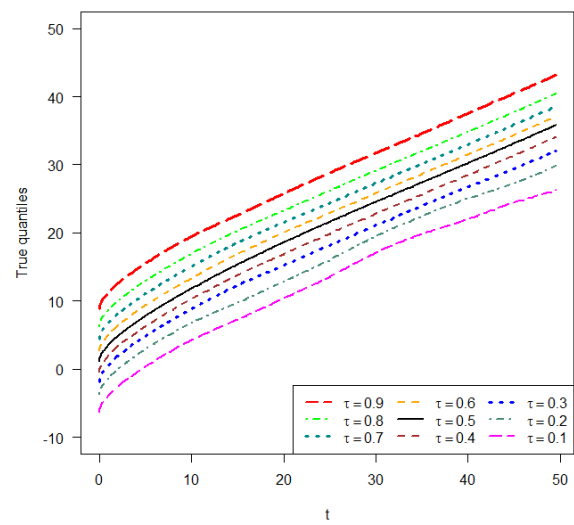

(c)

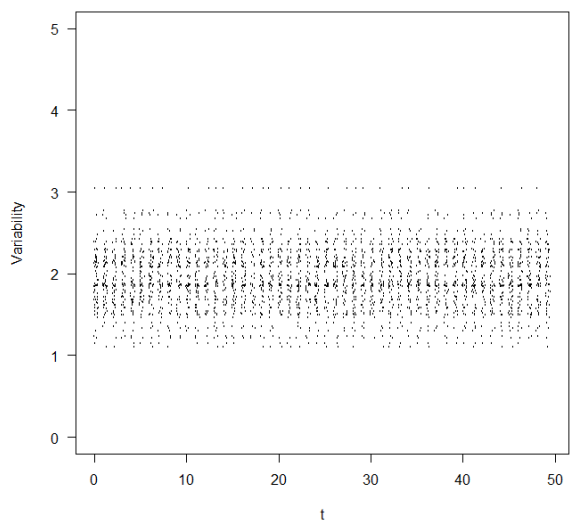

(b)

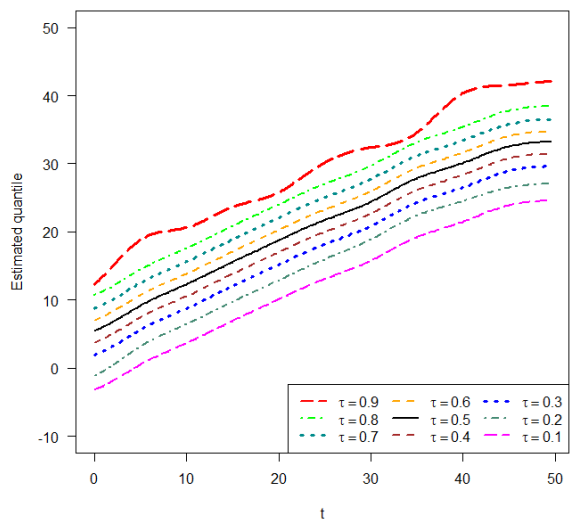

(d)

FIGURE 2: Setting 1. (a) True variability function; (b) Estimated variability function based on $V_{1}$; (c) True quantile curves; (d) Estimated quantile curves based on $V_{1}$.

Table 2 shows that $V_{0}$ is significantly worse than $V_{1}$, indicating that $\theta$ is different from zero or the variability function depends on the covariates via $\|\mathbf{X}(T)\|_{1}$. Furthermore, $V_{1}$ is not significantly worse than $V_{2}, V_{3}$ or $V_{4}$. Table 2 also reveals that $V_{0}$ is not significantly worse than $V_{5}$ and that $V_{5}$ is worse than $V_{2}, V_{3}$ or $V_{4}$. This indicates that $V_{5}$ is also worse than $V_{1}$. This result coincides with the simulation result given in Figure 1 (a). Similar results are obtained when the SNR is reduced to 4 or 0.5 . 
TABLE 2: Proportion of significant tests for Setting 1.

\begin{tabular}{|c|c|c|c|}
\hline \multirow[t]{2}{*}{ Comparison } & \multicolumn{3}{|c|}{ Proportion } \\
\hline & $S N R \approx 7$ & $S N R \approx 4$ & $S N R \approx 0.5$ \\
\hline$V_{0}$ vs $V_{1}$ & 1.00 & 1.00 & 1.00 \\
\hline$V_{1}$ vs $V_{2}$ & 0.02 & 0.00 & 0.00 \\
\hline$V_{1}$ vs $V_{3}$ & 0.01 & 0.00 & 0.01 \\
\hline$V_{1}$ vs $V_{4}$ & 0.02 & 0.02 & 0.02 \\
\hline$V_{0}$ vs $V_{5}$ & 0.02 & 0.00 & 0.01 \\
\hline$V_{5}$ vs $V_{2}$ & 1.00 & 1.00 & 1.00 \\
\hline$V_{5}$ vs $V_{3}$ & 1.00 & 1.00 & 1.00 \\
\hline$V_{5}$ vs $V_{4}$ & 1.00 & 1.00 & 1.00 \\
\hline
\end{tabular}

\subsection{Setting 4}

The data sets are simulated from Setting $4 . V_{4}$ has the best performance in estimating the quantiles as well as the variability function (see Figures 3 (a) and (b)). From Figure S3 of the Supplementary Material, it is clear that the true variability and quantile functions are estimated quite well using $V_{4}$.

Using the likelihood-ratio-type test, $V_{4}$ is compared to the other models. Table 3 shows that all the other models are worse than $V_{4}$ with power equal to one. This was expected since $V_{4}$ is the most flexible model. This coincides with the simulation result given in Figure 3. Table 3 also shows that when we reduce the SNR to 4 or 2, our test still maintains its power. 


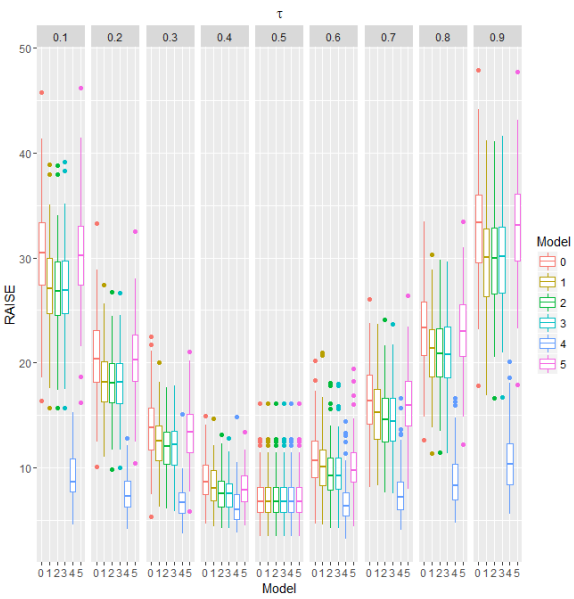

(a)

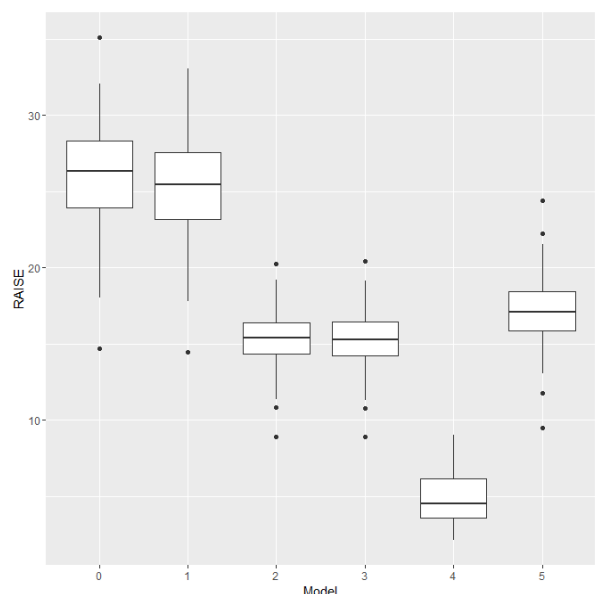

(b)

FIGURE 3: Setting 4. Boxplots of (a) $\operatorname{RAISE}\left(\hat{q}_{\tau}^{(s)}\right)$; (b) $\operatorname{RAISE}\left(\hat{V}^{(s)}\right)$

TABLE 3: Proportion of significant tests for Setting 4.

\begin{tabular}{lccc} 
Comparison & \multicolumn{3}{c}{ Proportion } \\
\cline { 2 - 4 } & $S N R \approx 7$ & $S N R \approx 4$ & $S N R \approx 2$ \\
$V_{0}$ vs $V_{4}$ & 1.00 & 1.00 & 1.00 \\
$V_{1}$ vs $V_{4}$ & 1.00 & 1.00 & 1.00 \\
$V_{2}$ vs $V_{4}$ & 1.00 & 1.00 & 1.00 \\
$V_{3}$ vs $V_{4}$ & 1.00 & 1.00 & 1.00 \\
$V_{5}$ vs $V_{4}$ & 1.00 & 1.00 & 1.00 \\
\hline
\end{tabular}

\section{REAL DATA EXAMPLE}

In this section, the estimation and testing procedures developed in Sections 3 and 4 are applied to a data set which is a subset of the Multicenter AIDS cohort study. The data contain repeated mea- 
surements of physical examinations and CD4 percentages of 283 homosexual men who became HIV-positive between 1984 and 1991. Each individual has a different number of measurements ranging from 1 to 14 , with median equals to 6 , due to missing of their appointments and the random HIV infection moments. There are 59 distinct time points in total. More details on the data can be found in Kaslow et al. (1987).

The covariates are $X^{(1)}$ : the smoking status ( 1 or 0 according to whether the individual ever or never smoked cigarettes), $X^{(2)}$ : age at HIV infection, and $X^{(3)}$ : the pre-infection CD4 percentage of the individual. The response $Y\left(t_{i j}\right)$ is the CD4 percentage at time $t_{i j}$, where $t_{i j}$ denotes the time in years of the $j$ th measurement on the $i$ th individual after HIV infection. HIV attacks the CD4 cells. The CD4 percentage reflects an individual's immune system status, the lower the CD4 percentage the weaker the immune system and the more vulnerable an individual is to infections. Hence, it is of interest for investigating the evolvement of an HIV/AIDS infection over time.

The simple heteroscedastic model has been previously used (by Andriyana, Gijbels, \& Verhasselt (2017)) with these data and the validity of the two assumptions of the AHe approach checked (and fulfilled). To analyze the data set, B-splines of degree three with 11 equidistant knots in the time interval and differencing of order one are used. The covariates are standardized as in Section 5. All tests are done at the 5\% level of significance.

As discussed in Section 4, several comparisons are performed using the likelihood-ratio-type test with $\mathrm{B}=200$ bootstrap samples. Table 4 shows that $V_{0}$ and $V_{1}$ are significantly worse than $V_{4}$. But, $V_{5}, V_{2}$ and $V_{3}$ are not significantly worse than $V_{4}$. Hence, for this data set the simple heteroscedastic model $\left(V_{5}\right)$ is recommended. The estimated variability functions for $V_{0}, V_{4}$ and $V_{5}$ are presented in Figure 4 (a). By using $V_{5}$, we see that the variability function varies with time. Furthermore, the estimated variability function of $V_{5}$ is located around the middle of the estimated variability function of $V_{4}$. The estimated function $\hat{\gamma}(T)$ in the variability function based 
on $V_{4}$ is given in Figure 4 (b). The estimators of the other coefficient functions in the variability function are approximately constant and equal to $-0.2270,-0.0078$ and 0.0147 for smoking status, age and pre-infection CD4 respectively (with only small deviations from a constant of magnitude $10^{-12}, 10^{-14}$ and $10^{-14}$, respectively).

Another example is available in the Supplementary Material (Section S3).

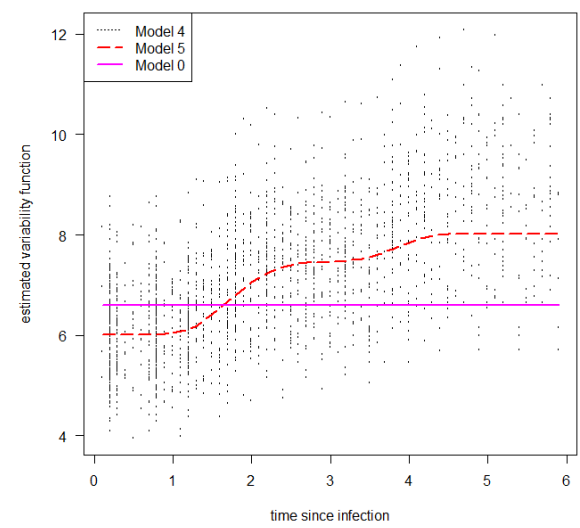

(a)

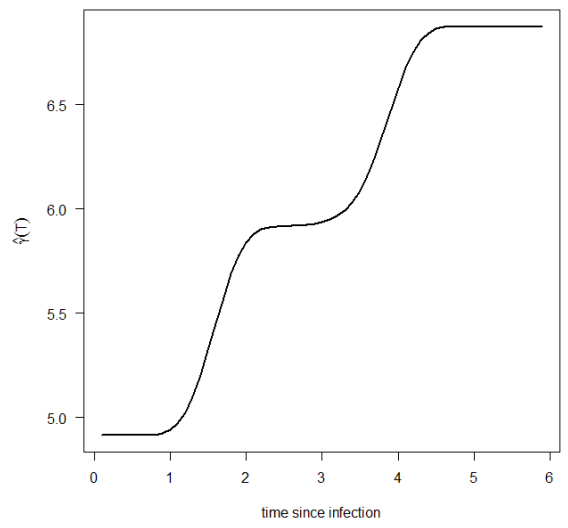

(b)

FIGURE 4: CD4 data. (a) Estimated variability functions; (b) $\hat{\gamma}(T)$ based on $V_{4}$.

TABLE 4: p-values for CD4 data.

$\begin{array}{lccccc}\text { Comparison } & V_{0} \text { vs } V_{4} & V_{1} \text { vs } V_{4} & V_{5} \text { vs } V_{4} & V_{2} \text { vs } V_{4} & V_{3} \text { vs } V_{4} \\ \text { p-value } & 0.0450 & 0.0400 & 0.1100 & 0.0950 & 0.1700\end{array}$




\section{DISCUSSION}

Various structures of the variability function are investigated. An estimation procedure for these functions is introduced and we show its consistency for an increasing number of knots. The simulation results show that the nesting structure of the different variability models is respected by the proposed estimation method. For instance, as is observed from simulation Setting 2 (in the Supplementary Material), $V_{2}$ out-performs $V_{0}, V_{1}$ and $V_{5}$ and has an equivalent performance when compared to the more complicated $V_{3}$ and $V_{4}$. The likelihood-ratio-type test confirms this finding.

Hypothesis testing is also performed using $B=99$ bootstrap samples for the simulation settings and the real data sets (not presented). Similar results are obtained using $B=200$ bootstrap samples for each setting. Although the likelihood-ratio-type test has good performance in the simulation studies, it is of interest to prove the consistency of the test. This consistency relies on the consistency of the estimators of the parameters as well as on the consistency of the bootstrap procedure. This is beyond the scope of this paper and is left for future research.

The variability structure $V_{4}$ is the most flexible modeling structure depending on the covariates. Hence, based on this model, one can do variable selection or test the constancy of the varying coefficient functions using the likelihood-ratio-type test.

In this paper we consider an exponential type of variability function to be the full model. Alternatively, one could also consider a single-index type of modeling (e.g. Wu, Yu, \& Yu, 2010; Lian, Liang, \& Carroll, 2015) where the variability function is $V(\mathbf{X}(T), T)=g\left(\mathbf{X}^{\prime}(T) \boldsymbol{\theta}(T)\right)$, with an unknown link function $g() \geq$.0 . To estimate the variability function we would then need to go through two steps: in the first step the single index $X^{\prime}(T) \theta(T)$ would be estimated with $g$ assumed to be approximatively linear, and in the second step the unknown link function estimated. 


\section{Acknowledgement}

The authors gratefully acknowledge support from the Interuniversity Attraction Poles Research Network P7/06 of the Belgian State (Belgian Science Policy). I. Gijbels acknowledges support from the Katholieke Universiteit Leuven Research Council. A. Verhasselt acknowledges support from the Research Foundation Flanders (Fonds Wetenschappelijk Onderzoek). M.A. Ibrahim and A. Verhasselt acknowledge support from the Special Research Fund (Bijzonder Onderzoeksfonds) of Hasselt University. The infrastructure of the Flemish Supercomputer Center (Vlaams Supercomputer Centrum), funded by the Hercules Foundation and the Flemish Government department of Economy, Science \& Innovation, was used for the simulations. We would like to thank the Editor, an associate editor and two reviewers for their constructive comments that led to a further improvement of this article.

\section{BIBLIOGRAPHY}

Andriyana, Y. (2015). P-splines quantile regression in varying coefficient models. $\mathrm{PhD}$ Dissertation $\mathrm{KU}$ Leuven, Belgium. ISBN 978-90-8649-791-1.

Andriyana, Y. \& Gijbels, I. (2017). Quantile regression in heteroscedastic varying coefficient models. AStA Advances in Statistical Analysis, 101, 151-176.

Andriyana, Y., Gijbels, I., \& Verhasselt, A. (2014). P-splines quantile regression estimation in varying coefficient models. Test, 23, 153-194.

Andriyana, Y., Gijbels, I., \& Verhasselt, A. (2017). Quantile regression in varying-coefficient models: noncrossing quantile curves and heteroscedasticity. Statistical Papers, to appear.

DOI : $10.1007 / \mathrm{s} 00362-016-0847-7$

Antoniadis, A., Gijbels, I., \& Verhasselt, A. (2012). Variable selection in varying coefficient models using P-splines. Journal of Computational and Graphical Statistics, 21, 638-661.

Bollaerts, K., Eilers, P., \& Aerts, M. (2006). Quantile regression with monotonicity restrictions using psplines and the 11-norm. Statistical Modelling, 6, 189-207. 
Cai, Z. \& Xu, X. (2008). Nonparametric quantile estimations for dynamic smooth coefficient models. Journal of the American Statistical Association, 103, 1595-1608.

Chaudhuri, P. (1991). Global nonparametric estimation of conditional quantile functions and their derivatives. Journal of Multivariate Analysis, 39, 246-269.

Claeskens, G., Krivobokova, T. \& Opsomer, J. (2009). Asymptotic properties of penalize spline estimators. Biometrika, 96, 529-544.

Davidian, M. \& Giltinan, D. (1995). Nonlinear models for repeated measurement data, Chapman \& Hall/CRC, New York.

Eilers, P.H.C. \& Marx, B.D. (1996). Flexible smoothing with B-splines and penaltie. Statistical Science, 11, $89-121$.

Fortin, M., Daigle, G., Ung, C., Bégin, J., \& Archambault, L. (2007). A variance-covariance structure to take into account repeated measurements and heteroscedasticity in growth modeling. European Journal of Forest Research, 126, 573-585.

Gijbels, I. \& Verhasselt, A. (2010). P-splines regression smoothing and difference type of penalty. Statistics and Computing, 20, 499-511.

Hastie, T. \& Tibshirani, R. (1993). Varying-coefficient models. Journal of the Royal Statistical Society. Series $B, 55,757-796$.

He, X. (1997). Quantile curves without crossing. The American Statistician, 51, 186-192.

He, X., Ng, P., \& Portnoy, S. (1998). Bivariate quantile smoothing splines. Journal of the Royal Statistical Society. Series B, 60, 537-550.

He, X. \& Shi, P. (1994). Convergence rate of b-spline estimators of nonparametric conditional quantile functions. Journal of Nonparametric Statistics, 3, 299-308.

Honda, T. (2004). Quantile regression in varying coefficient models. Journal of Statistical Planning and Inference, 121, 113-125. 
Kaslow, R., Ostrow, D., Detels, R., Phair, J., Polk, B., \& Rinaldo, C. (1987). The multicenter aids cohort study: rationale, organization, and selected characteristics of the participants. American Journal of Epidemiology, 126, 310-318.

Kim, M. (2007). Quantile regression with varying coefficients. The Annals of Statistics, 35, 92-108.

Koenker, R. (2005). Quantile regression. Cambridge university press, New York.

Koenker, R. \& Bassett, G. (1978). Regression quantiles. Econometrica, 46, 33-50.

Lian, H., Liang, H., \& Carroll, R. J. (2015). Variance function partially linear single-index models. Journal of the Royal Statistical Society: Series B (Statistical Methodology), 77, 171-194.

Noh, H., Chung, K., \& Van Keilegom, I. (2012). Variable selection of varying coefficient models in quantile regression. Electronic Journal of Statistics, 6, 1220-1238.

Schumaker, L. (1981). Spline functions: basic theory, Wiley, New York.

Stone, C. (1982). Optimal global rates of convergence for nonparametric regression. The Annals of Statistics, 10, 1040-1053.

Tang, Y., Wang, H., \& Zhu, Z. (2013). Variable selection in quantile varying coefficient models with longitudinal data. Computational Statistics \& Data Analysis, 57, 435-449.

Van Keilegom, I. \& Wang, L. (2010). Semiparametric modeling and estimation of heteroscedasticity in regression analysis of cross-sectional data. Electronic Journal of Statistics, 4, 133-160.

Wu, T. Z., Yu, K., \& Yu, Y. (2010). Single-index quantile regression. Journal of Multivariate Analysis, 101, $1607-1621$.

\section{APPENDIX}

$\underline{\text { Appendix A: Definitions and assumptions }}$

Definition 1 For two sequences of nonnegative real numbers $a_{n}$ and $b_{n}, a_{n} \sim b_{n}$ denotes that there are constants $0<a<A<\infty$ such that $a \leq a_{n} / b_{n} \leq$ A for all $n$. 
Definition 2 The collection of all functions on $\mathcal{T}$ for which the lth order derivative satisfies the Hölder condition of order $\mathfrak{H}$ with $r=l+\mathfrak{H}$ is denoted by $\mathcal{H}_{r}$. That is, for each $h \in \mathcal{H}_{r}$, $\left|h^{(l)}(s)-h^{(l)}(t)\right| \leq c|s-t|^{\mathfrak{H}}$ for any $s, t \in \mathcal{T}$, and a positive constant $c$.

We introduce additional notation.

$$
\begin{array}{ll}
R_{i j}=\sum_{k=0}^{p}\left(\tilde{\theta}_{k}\left(t_{i j}\right)-\theta_{k}\left(t_{i j}\right)\right)\left|X^{(k)}\left(t_{i j}\right)\right| ; & m_{\max }^{\mathrm{v}}=\max \left(m_{0}^{\mathrm{v}}, \ldots, m_{p}^{\mathrm{v}}\right) ; \\
\left(\mathbf{U}^{\mathrm{v}}\right)^{(k)}(T)=\left|X^{(k)}(T)\right| \mathbf{B}_{k}^{\mathrm{v}}\left(T, \nu_{k}^{\mathrm{v}}\right) ; & \lambda_{\max }^{\mathrm{v}}=\max \left(\lambda_{0}^{\mathrm{v}}, \ldots, \lambda_{p}^{\mathrm{v}}\right) ; \\
\mathbf{U}^{\mathrm{v}}(T)=\left(\left(\mathbf{U}^{\mathrm{v}}\right)^{(1)}(T)^{\prime}, \ldots,\left(\mathbf{U}^{\mathrm{v}}\right)^{(p)}(T)^{\prime}\right)^{\prime} ; & m_{t o t}^{\mathrm{v}}=m_{0}^{\mathrm{v}}+m_{1}^{\mathrm{v}}+\ldots+m_{p}^{\mathrm{v}} ; \\
\mathbf{Z}=\left(\mathbf{U}^{\mathrm{v}}\left(t_{11}\right), \ldots, \mathbf{U}^{\mathrm{v}}\left(t_{1 N_{1}}\right), \ldots, \mathbf{U}^{\mathrm{v}}\left(t_{n 1}\right), \ldots, \mathbf{U}^{\mathrm{v}}\left(t_{n N_{n}}\right)\right) ; \\
\mathbf{H}^{2}=\mathbf{Z}^{\prime} \mathbf{Z} ; & \mathbf{z}_{i j}=\mathbf{H}^{+} \mathbf{U}^{\mathrm{v}}\left(t_{i j}\right) ; \\
\hat{\boldsymbol{\alpha}}^{\mathrm{v}}=\left(\left(\hat{\boldsymbol{\alpha}}_{0}^{\mathrm{v}}\right)^{\prime},\left(\hat{\boldsymbol{\alpha}}_{1}^{\mathrm{v}}\right)^{\prime}, \ldots,\left(\hat{\boldsymbol{\alpha}}_{p}^{\mathrm{v}}\right)^{\prime}\right)^{\prime} ; & \hat{\boldsymbol{\theta}}^{*}=\mathbf{H} \hat{\boldsymbol{\alpha}}^{\mathrm{v}} ; \\
\tilde{\boldsymbol{\alpha}}^{\mathrm{v}}=\left(\left(\tilde{\boldsymbol{\alpha}}_{0}^{\mathrm{v}}\right)^{\prime},\left(\tilde{\boldsymbol{\alpha}}_{1}^{\mathrm{v}}\right)^{\prime}, \ldots,\left(\tilde{\boldsymbol{\alpha}}_{p}^{\mathrm{v}}\right)^{\prime}\right)^{\prime} ; & \tilde{\boldsymbol{\theta}}^{*}=\mathbf{H}^{\mathrm{v}} ; \\
\epsilon_{i j}^{\mathrm{v}}=\epsilon^{\mathrm{v}}\left(t_{i j}\right) ; & N=\sum_{i=1}^{n} N_{i} ;
\end{array}
$$

where $\mathbf{H}^{+}$denotes the Moore inverse of $\mathbf{H}$, and for a vector $\mathbf{u},\|\mathbf{u}\|_{2}$ denotes its Euclidean norm.

\section{Assumptions:}

B1. For $k=0,1, \ldots, p, \beta_{k} \in \mathcal{H}_{r_{k}}$, for some $r_{k}>1 / 2$. Let $c_{k}$ denote the Hölder constant for the $k t h$ varying coefficient function $\beta_{k}$.

B2. $\lim \sup _{n \rightarrow \infty}\left(\frac{u_{\max }}{u_{\min }}\right)<\infty$, with $u_{\min }=\min \left(u_{0}, \ldots, u_{p}\right)$.

B3. The error terms $\epsilon\left(t_{i j}\right)$ and $\epsilon\left(t_{l z}\right)$ are independent for all $i \neq l$ and for all $j$ and $z$, with $i, l \in$ $\{1, \ldots, n\}, j \in\left\{1, \ldots, N_{i}\right\}$ and $z \in\left\{1, \ldots, N_{l}\right\}$. The distributions of $\left(\epsilon\left(t_{i 1}\right), \ldots, \epsilon\left(t_{i N_{i}}\right)\right)$ are the same for all $i$. The density function of $\epsilon\left(t_{i j}\right)$ given $\left(\mathbf{X}\left(t_{i j}\right), t_{i j}\right), f_{\epsilon}$, is continuous at zero and bounded away from zero and infinity.

B4. $\lim _{n \rightarrow \infty} u_{\max } n^{\delta^{M}-1}=0$ for some $\delta^{M} \in(0,1)$.

B5. $u_{\max } \sim n^{1 /\left(2 r_{\min }+1\right)}$, where $r_{\min }=\min \left(r_{0}, \ldots, r_{p}\right)$. 
B6. The conditional distribution of $T$, given $\mathbf{X}=\mathbf{x}$ has a bounded density $f_{T \mid \mathbf{X}}: 0<b \leq$ $f_{T \mid \mathbf{X}}(t \mid \mathbf{x}) \leq B<\infty$, uniformly in $\mathbf{x}$ and $t$ for some positive constants $b$ and $B$.

B7. $E\left(X^{(k)}(T) \mid T\right)=0$ and $P\left(\left|X^{(k)}(T)\right|<M\right)=1$ for some $M<\infty, k=1, \ldots, p$, and there exist two positive definite matrices $\boldsymbol{\Sigma}_{1}$ and $\boldsymbol{\Sigma}_{2}$ such that $\boldsymbol{\Sigma}_{1} \leq \operatorname{Var}(\mathbf{X}(T) \mid T) \leq \boldsymbol{\Sigma}_{2}$ uniformly in $T$, where $\operatorname{Var}(\mathbf{X}(T) \mid T)$ denotes the conditional covariance matrix of $\mathbf{X}(T)$ given $T$.

C1. For $k=0,1, \ldots, p, \theta_{k} \in \mathcal{H}_{r_{k}^{\mathrm{v}}}$, for some $r_{k}^{\mathrm{v}}>1 / 2$. Let $c_{k}^{\mathrm{v}}$ denote the Hölder constant for the $k t h$ varying coefficient function $\theta_{k}$.

C2. Let $\epsilon^{\mathrm{v}}(T)=\ln (|\epsilon(T)|)$. The error terms $\epsilon^{\mathrm{v}}\left(t_{i j}\right)$ and $\epsilon^{\mathrm{v}}\left(t_{l z}\right)$ are independent for all $i \neq l$ and for all $j$ and $z$, with $i, l \in\{1, \ldots, n\}, j \in\left\{1, \ldots, N_{i}\right\}$ and $z \in\left\{1, \ldots, N_{l}\right\}$. The distribution of $\epsilon^{\mathrm{v}}\left(t_{i j}\right)$, given $\left(\mathbf{X}\left(t_{i j}\right), t_{i j}\right)$, has a density function $f_{\epsilon^{\mathrm{v}}}$ which is continuous at zero with $f_{\epsilon} \mathrm{v}(0)>0$.

C3. $\lim _{n \rightarrow \infty}\left(u_{\max }^{\mathrm{v}}\right)^{2} n^{\delta^{\mathrm{v}}-1}=0$ for some $\left.\delta^{\mathrm{v}} \in\right] 0,1\left[\right.$, where $u_{\max }^{\mathrm{v}}=\max \left(u_{0}^{\mathrm{v}}, \ldots, u_{p}^{\mathrm{v}}\right)$.

C4. $u_{\max }^{\mathrm{v}} \sim n^{1 /\left(2 r_{\min }^{\mathrm{v}}+1\right)}$, with $r_{\min }^{\mathrm{v}}=\min \left(r_{0}^{\mathrm{v}}, \ldots, r_{p}^{\mathrm{v}}\right)$.

C5. $\lim \sup _{n \rightarrow \infty}\left(\frac{u_{\max }^{\mathrm{v}}}{u_{\min }^{\mathrm{V}}}\right)<\infty$, with $u_{\min }^{\mathrm{v}}=\min \left(u_{0}^{\mathrm{v}}, \ldots, u_{p}^{\mathrm{v}}\right)$.

C6. $\theta_{k}(t)$ is $\nu_{k}^{\mathrm{v}}-1$ times continuously differentiable on $t \in \mathcal{T}$, with $\nu_{k}^{\mathrm{v}} \geq 1$.

\section{Appendix B: Preliminary lemmas}

The proof of Theorem 2 is based on the following lemmas.

Lemma 1 is a straightforward application of Corollary 6.21 in Schumaker (1981).

Lemma 1 Assume $\mathbf{C 1}$ holds. For some constant $W_{1, k}$ that depends only on $\nu_{k}^{V}$ and $c_{k}^{V}$, there exists a spline coefficient vector $\tilde{\boldsymbol{\alpha}}_{k}^{V}$ such that, $\sup _{t \in \mathcal{T}}\left|\left(\tilde{\boldsymbol{\alpha}}_{k}^{V}\right)^{\prime} \mathbf{B}_{k}^{V}\left(t, \nu_{k}^{V}\right)-\theta_{k}(t)\right| \leq W_{1, k}\left(u_{k}^{V}\right)^{-r_{k}^{V}}$.

The following two lemmas are derived from He \& Shi (1994) (Lemmas 3.4 and 3.5, respectively). 
Lemma 2 Under $\mathbf{C 3}$, there exists a positive constant $W_{2, k}$ depending only on $b, B, \nu_{k}^{V}$ and $c_{k}^{V}$ such that, except on an event whose probability tends to zero with $n$, for all $\hat{\theta}_{k}$ and $\theta_{k} \in \mathcal{H}_{r_{k}^{V}}$,

$$
\left\|\hat{\theta}_{k}-\theta_{k}\right\|_{2}^{2} \leq W_{2, k}\left\{\left(u_{\max }^{V}\right)^{-2 r_{\min }^{V}}+\frac{1}{n} \sum_{i=1}^{n} \frac{1}{N_{i}} \sum_{j=1}^{N_{i}}\left(\hat{\theta}_{k}\left(t_{i j}\right)-\theta_{k}\left(t_{i j}\right)\right)^{2}\right\}
$$

Lemma 3 There exists a positive constant $W_{3, k}$ such that, for all $\hat{\theta}_{k}$ and $\theta_{k} \in \mathcal{H}_{r_{k}^{V}}$,

$$
\left\|\hat{\theta}_{k}^{(j)}-\theta_{k}^{(j)}\right\|_{2}^{2} \leq W_{3, k}\left\{\left(u_{\max }^{V}\right)^{2\left(j-r_{\min }^{V}\right)}+\left(u_{\max }^{V}\right)^{2 j}\left\|\hat{\theta}_{k}-\theta_{k}\right\|_{2}^{2}\right\}, \quad j=0,1, \ldots, \nu_{k}^{V}-1
$$

Furthermore, we need the following lemma.

Lemma 4 Assume B6, B7, C1, C2 and C4 hold, and $\boldsymbol{\xi} \in \mathbb{R}^{m_{\text {tot }}^{V}}$.

(a) For any sequence $\left\{L_{n}\right\}$ satisfying $1 \leq L_{n} \leq\left(u_{\max }^{V}\right)^{\delta_{0} / 10}$, for some $0<\delta_{0}<\left(r_{\min }^{V}-\right.$ $1 / 2) /\left(2 r_{\min }^{V}+1\right)$,

$$
\begin{aligned}
& \sup _{\|\boldsymbol{\xi}\|_{2} \leq 1}\left(u_{\max }^{V}\right)^{-1} \mid \sum_{i=1}^{n} \frac{1}{N_{i}} \sum_{j=1}^{N_{i}}\left[\rho_{0.5}\left\{\epsilon_{i j}^{V}-L_{n}\left(u_{\max }^{V}\right)^{1 / 2} \mathbf{z}_{i j}^{\prime} \boldsymbol{\xi}-R_{i j}\right\}-\rho_{0.5}\left\{\epsilon_{i j}^{V}-R_{i j}\right\}\right. \\
& \left.+L_{n}\left(u_{\max }^{V}\right)^{1 / 2} \mathbf{z}_{i j}^{\prime} \boldsymbol{\xi}\left(0.5-I\left(\epsilon_{i j}^{V}<0\right)\right)-E\left[\rho_{0.5}\left\{\epsilon_{i j}^{V}-L_{n}\left(u_{\max }^{V}\right)^{1 / 2} \mathbf{z}_{i j}^{\prime} \boldsymbol{\xi}-R_{i j}\right\}-\rho_{0.5}\left\{\epsilon_{i j}^{V}-R_{i j}\right\}\right]\right] \mid=o_{p}(1),
\end{aligned}
$$

where $E$ is the conditional expectation given $\left(\mathbf{X}\left(t_{i j}\right), t_{i j}\right)$ for $i=1, \ldots, n ; j=1, \ldots, N_{i}$.

(b) For any $\omega>0$, there exists $L$ (sufficiently large) such that as $n \rightarrow \infty$,

$$
\begin{array}{r}
P\left\{( u _ { \operatorname { m a x } } ^ { V } ) ^ { - 1 } \left(\inf _{\|\boldsymbol{\xi}\|_{2}=1} \sum_{i=1}^{n} \frac{1}{N_{i}} \sum_{j=1}^{N_{i}} E\left[\rho_{0.5}\left\{\epsilon_{i j}^{V}-L\left(u_{\max }^{V}\right)^{1 / 2} \mathbf{z}_{i j}^{\prime} \boldsymbol{\xi}-R_{i j}\right\}-\rho_{0.5}\left\{\epsilon_{i j}^{V}-R_{i j}\right\}\right]\right.\right. \\
\left.\left.-L\left(u_{\max }^{V}\right)^{1 / 2}\left\|\sum_{i=1}^{n} \frac{1}{N_{i}} \sum_{j=1}^{N_{i}} \mathbf{z}_{i j}\left(0.5-I\left(\epsilon_{i j}^{V}<0\right)\right)\right\| \|_{2}\right)>1\right\}>1-\omega .
\end{array}
$$

The proof of Lemma 4 follows similar lines to those of Lemmas 3.2 and 3.3 in He \& Shi (1994). The difference arises from the calculations of (3.7) and (3.10) in He \& Shi (1994); here the correlation of observations within subject $i$ has to be taken into account. However, the goal is to find an upper bound for the aforementioned two expressions and since the correlation is at most one, we still attain the same upper bounds for the expressions. Therefore, we omit the proof.

DOI: 
Lemma 5 Assume B6, B7, C1, C2 and C4 hold. Furthermore, assume that

$\left(u_{\max }^{V}\right)^{3 / 4} \lambda_{\max }^{V} n^{-1 / 2} \rightarrow 0$ as $n$ tends to $\infty$

Then, $\sum_{i=1}^{n} \frac{1}{N_{i}} \sum_{j=1}^{N_{i}}\left(\hat{\theta}_{k}\left(t_{i j}\right)-\tilde{\theta}_{k}\left(t_{i j}\right)\right)^{2}=O_{p}\left(u_{\max }^{V}\right)$.

Proof of Lemma 5. The proof of Lemma 5 is along the same lines as the proof of Theorem 2.1 of He \& Shi (1994). Using Lemma 4, for any $\omega>0$, there exists $L$ such that as $n \rightarrow \infty$,

$P\left\{\left(u_{\max }^{\mathrm{v}}\right)^{-1}\left(\inf _{\|\boldsymbol{\xi}\|_{2}=L\left(u_{\max }^{\mathrm{V}}\right)^{1 / 2}} \sum_{i=1}^{n} \frac{1}{N_{i}} \sum_{j=1}^{N_{i}} \rho_{0.5}\left\{\epsilon_{i j}^{\mathrm{v}}-\mathbf{z}_{i j}^{\prime} \boldsymbol{\xi}-R_{i j}\right\}-\sum_{i=1}^{n} \frac{1}{N_{i}} \sum_{j=1}^{N_{i}} \rho_{0.5}\left\{\epsilon_{i j}^{\mathrm{v}}-R_{i j}\right\}\right)>1\right\}>1-\omega$

With probability one, we have

$$
\sum_{i=1}^{n} \frac{1}{N_{i}} \sum_{j=1}^{N_{i}} \rho_{0.5}\left\{\epsilon_{i j}^{\mathrm{v}}-\mathbf{z}_{i j}^{\prime}\left(\hat{\boldsymbol{\theta}}^{*}-\tilde{\boldsymbol{\theta}}^{*}\right)-R_{i j}\right\}=\inf _{\|\boldsymbol{\xi}\|_{2} \in \mathbb{R}^{m_{\text {tot }}^{\mathrm{v}}}} \sum_{i=1}^{n} \frac{1}{N_{i}} \sum_{j=1}^{N_{i}} \rho_{0.5}\left\{\epsilon_{i j}^{\mathrm{v}}-\mathbf{z}_{i j}^{\prime} \boldsymbol{\xi}-R_{i j}\right\} .
$$

From the proof of Lemma 2.3 in Andriyana, Gijbels, \& Verhasselt (2014), using $\| \hat{\boldsymbol{\theta}}^{*}-$ $\tilde{\boldsymbol{\theta}}^{*} \|_{2}=L\left(u_{\max }^{\mathrm{v}}\right)^{1 / 2}$ and the matrix-representation $\mathbf{D}_{k}^{d_{k}^{\mathrm{V}}}$ of the differencing operator $\Delta^{d_{k}^{\mathrm{v}}}$, we have

$$
\sum_{k=0}^{p} \lambda_{k}^{\mathrm{v}}\left\|\mathbf{D}_{k}^{d_{k}^{\mathrm{v}}} \hat{\boldsymbol{\alpha}}_{k}^{\mathrm{v}}\right\|_{1}-\sum_{k=0}^{p} \lambda_{k}^{\mathrm{v}}\left\|\mathbf{D}_{k}^{d_{k}^{\mathrm{V}}} \tilde{\boldsymbol{\alpha}}_{k}^{\mathrm{v}}\right\|_{1}=o_{p}\left(u_{\max }^{\mathrm{v}}\right)
$$

where for $d_{k}^{\mathrm{v}}=1, \mathbf{D}_{k}^{d_{k}^{\mathrm{v}}}=\left(\begin{array}{cccccccc}1 & -1 & 0 & 0 & \ldots & 0 & 0 & 0 \\ 0 & 1 & -1 & 0 & \ldots & 0 & 0 & 0 \\ \vdots & \vdots & \vdots & \vdots & \vdots & \vdots & \vdots & \vdots \\ 0 & 0 & 0 & 0 & \ldots & 0 & 1 & -1\end{array}\right) \in \mathbb{R}^{\left(m_{k}^{\mathrm{v}}-d_{k}^{\mathrm{V}}\right) \times m_{k}^{\mathrm{v}}}$.

By Equations (A.2), (A.3) and since

$$
\sum_{k=0}^{p} \sum_{i=1}^{n} \sum_{j=1}^{N_{i}}\left(\hat{\theta}_{k}\left(t_{i j}\right)\left|X^{(k)}\left(t_{i j}\right)\right|-\tilde{\theta}_{k}\left(t_{i j}\right)\left|X^{(k)}\left(t_{i j}\right)\right|\right)^{2}=\left\|\hat{\boldsymbol{\theta}}^{*}-\tilde{\boldsymbol{\theta}}^{*}\right\|_{2}^{2}
$$


Equation (A.1) implies that

$$
\begin{array}{r}
P\left(\sum_{k=0}^{p} \sum_{i=1}^{n} \sum_{j=1}^{N_{i}}\left(\hat{\theta}_{k}\left(t_{i j}\right)\left|X^{(k)}\left(t_{i j}\right)\right|-\tilde{\theta}_{k}\left(t_{i j}\right)\left|X^{(k)}\left(t_{i j}\right)\right|\right)^{2} \leq L^{2} u_{\max }^{\mathrm{v}}\right) \\
=P\left(\left\|\hat{\boldsymbol{\theta}}^{*}-\tilde{\boldsymbol{\theta}}^{*}\right\|_{2} \leq L\left(u_{\max }^{\mathrm{v}}\right)^{1 / 2}\right)>1-\omega,
\end{array}
$$

which proves the lemma.

\section{Appendix C: Proof of Theorem 2}

Let us introduce additional notation: $\mathbf{B}_{k}^{\mathrm{v}}\left(T, \nu_{k}^{\mathrm{v}}\right)=\left(B_{k 1}^{\mathrm{v}}\left(T, \nu_{k}^{\mathrm{v}}\right), \ldots, B_{k m_{k}^{\mathrm{v}}}^{\mathrm{v}}\left(T, \nu_{k}^{\mathrm{v}}\right)\right)^{\prime}$ and $\tilde{\theta}_{k}(T)=\left(\tilde{\boldsymbol{\alpha}}_{k}^{\mathrm{v}}\right)^{\prime} \mathbf{B}_{k}^{\mathrm{v}}\left(T, \nu_{k}^{\mathrm{v}}\right)$, where the $\tilde{\boldsymbol{\alpha}}_{k}^{\mathrm{v}}$ are the coefficients of the best possible spline approximation of $\theta_{k}(T)$ as defined in Lemma 1.

Proof of Theorem 2. First, by the fact that $2 a b \leq a^{2}+b^{2}$ for any $(a, b) \in \mathbb{R}^{2}$, we have

$$
\begin{aligned}
& \frac{1}{n} \sum_{i=1}^{n} \frac{1}{N_{i}} \sum_{j=1}^{N_{i}}\left(\hat{\theta}_{k}\left(t_{i j}\right)-\theta_{k}\left(t_{i j}\right)\right)^{2} \\
& \quad \leq \frac{2}{n} \sum_{i=1}^{n} \frac{1}{N_{i}} \sum_{j=1}^{N_{i}}\left(\hat{\theta}_{k}\left(t_{i j}\right)-\tilde{\theta}_{k}\left(t_{i j}\right)\right)^{2}+2 \sum_{i=1}^{n} \frac{1}{N_{i}} \sum_{j=1}^{N_{i}}\left(\tilde{\theta}_{k}\left(t_{i j}\right)-\theta_{k}\left(t_{i j}\right)\right)^{2} .
\end{aligned}
$$

By Lemma 5, we have that

$$
\frac{2}{n} \sum_{i=1}^{n} \frac{1}{N_{i}} \sum_{j=1}^{N_{i}}\left(\hat{\theta}_{k}\left(t_{i j}\right)-\tilde{\theta}_{k}\left(t_{i j}\right)\right)^{2}=\frac{2}{n} O_{p}\left(u_{\max }^{\mathrm{v}}\right)=O_{p}\left(u_{\max }^{\mathrm{v}} n^{-1}\right) .
$$

By Lemma 1, we have that

$2 \sum_{i=1}^{n} \frac{1}{N_{i}} \sum_{j=1}^{N_{i}}\left(\tilde{\theta}_{k}\left(t_{i j}\right)-\theta_{k}\left(t_{i j}\right)\right)^{2} \leq 2\left(\max _{k=0, \ldots, p}\left(W_{1, k}\right)\right)^{2}\left(u_{\max }^{\mathrm{v}}\right)^{-2 r_{\min }^{\mathrm{v}}}=O_{p}\left(\left(u_{\max }^{\mathrm{v}}\right)^{-2 r_{\min }^{\mathrm{v}}}\right)$.

Combining Equations (A.4) and (A.5) leads to

$$
\begin{aligned}
\frac{1}{n} \sum_{i=1}^{n} \frac{1}{N_{i}} \sum_{j=1}^{N_{i}}\left(\hat{\theta}_{k}\left(t_{i j}\right)-\theta_{k}\left(t_{i j}\right)\right)^{2} & =O_{p}\left(u_{\max }^{\mathrm{v}} n^{-1}+\left(u_{\max }^{\mathrm{v}}\right)^{-2 r_{\min }^{\mathrm{v}}}\right) \\
& =O_{p}\left(n^{-2 r_{\min }^{\mathrm{v}} /\left(2 r_{\min }^{\mathrm{v}}+1\right)}\right) .
\end{aligned}
$$


Using Equation (A.6), Lemmas 2 and 3, the proof of our main result is now complete.

Received 21 November 2016

Accepted 10 September 2017 